Military Technical College, Kobry El-Kobbah, Cairo, Egypt $9^{\text {th }}$ International Conference On Aerospace Sciences \& Aviation Technology

\title{
PERFORMANCE OF FREQUENCY AGILE RADAR IN PRESENCE OF JAMMING SIGNAL
}

MEKHAIL Nabil G.; ELbarbary K. ", and ABDEL-LATIF Mohamed S.

\section{ABSTRACT}

In this paper, analysis of frequency agile radar (FAR) performance in presence of different jamming techniques will be presented. Many performance measures may be probability of false alarm improvement factor alarm $\left(\mathrm{P}_{\mathrm{fa}}\right)$, reduction of interference signals, and SNR performance in presence radar assumed to be a search radar, and the jamming types. The radar under consideration is detection for frequency radar, and the jammer is stand off jammer. The probability of case of presence of using frequency of only noise and the case of noise and jamming signal. Effect of jamming will be evaluated. Two jacM technique against the spot and barrage management of evaluated. Two jamming techniques are proposed for optimum frequency modulation jer power; namely the "partial band jamming" and "linear different jamming jamming" will be discussed. Comparison among the effect of discussed.

\section{KEY WORDS :}

Agile Radar, Jamming.

\footnotetext{
Egyptian Armed Forces
} 


\section{Introduction :}

Assuming that the radar observation time is equal to $N$ pulse repetition intervals each of duration $T_{R}$. At the end of the observation time, which is, $N T_{R}$, the radar is called on to decide: Is there only noise being received, or there is a signal being received with noise. Another important question is: how should the radar process the observed waveform $y(t)$ to obtain the best possible decision at time $N T_{R}$ ? The second question's answer defines the matched filter (MF) for the signal as an optimum filters for higher probability of detection.

Bayes's decision theory deals with correct and incorrect decisions that can be made in the detection process. Having an observation $\mathrm{y}(\mathrm{t})$ during time interval $\left[0, N T_{R}\right]$, two hypothesis can be defined

$$
\begin{aligned}
& H_{0}: y(t)=n_{r}(t), \text { Noise alone. } \\
& H_{1}: y(t)=s_{r}(t)+n_{r}(t), \text { Signal pluse noise. }
\end{aligned}
$$

It is assumed that the matched filter processing is implemented in video part after the receiver down conversion. However, the processor's job to determine if $s_{r}(t)$ is present or not, which is the detection process. In the figure (1), if the matched filter output $y_{1}(t)$ exceeds a threshold level $V, H_{1}$ is decided and if not, $H_{0}$ is decided. The threshold $V$ is expressed as

$$
V=\frac{N \sqrt{S N R}}{2}+\frac{1}{\sqrt{S N R}} \ln \left(V_{\tau}\right)
$$

Where $V_{T}$ is a detection threshold defined as a ratio between the probability of false alarm multiplied by its cost to the probability of missed detection multiplied by its cost and SNR is the signal to noise ratio.

As the integration period is $N T_{R}$, sampling can be done at the end of the target signal in the last (the Nth) period.

Frequency agile radar has main parameters, which is start frequency $f_{\text {star }}$, stop frequency $f_{\text {stop }}$, agile bandwidth $\beta_{t}$, total number of channels $v$, and minimum frequency separation between two successive frequencies $\Delta f$. So from the previous definitions the number of channels can be defined as $v=\beta_{t} / \Delta f$. 


\section{Performance of FAR Without Jamming}

System performance is determined, for a pulse-to-pulse frequency agile radar without jamming, by evaluating $y_{1}(t)$ in figure (1) at the desired sample time instant $\left(t_{0}\right)$. A single pulse is assumed $(N=1)$. Noting that:

$$
\begin{aligned}
& H_{1}: y(t)=s_{r}(t)+n_{w}(t) \\
& \because y_{1}\left(T_{R}\right)=\frac{2}{\sqrt{S N R} N_{o}} \int_{0}^{T_{r}} y(\tau) s_{r}(\tau) d \tau
\end{aligned}
$$

So $y_{1}\left(\right.$ at $\left.t=T_{R}\right)$ is Gaussian random variable with variance one. However, its mean is zero where $H_{0}$ hold and $\sqrt{S N R}$ for $H_{1}$. Since a false alarm occurs for $\mathrm{D} \geq 0$ and $H_{0}$ hold, its probability is

$$
P_{f a}=\int_{V}^{\infty} \frac{e^{-y_{1}^{2} / 2}}{\sqrt{2 \pi}} d y_{1}=Q(V)
$$

Where $\mathrm{Q}(V)$ is the $\mathrm{Q}$ function. In similar manner, $y_{1}$ is Gaussian with mean $\sqrt{S N R}$ and variance 1 when a target is present. The Detection probability is [1]

$$
P_{d}=\int_{V}^{\infty} \frac{e^{-\left(r_{1}-\sqrt{S N R}\right)^{2} / 2}}{\sqrt{2 \pi}} d y_{1}=Q(V-\sqrt{S N R})
$$

Figure (2) used the approximation to determine $V$ for various $P_{f a}$. Similarly it was used to generate figure (3) which describes how $P_{d}$ varies with $S N R$ for frequency agile radar without jamming. As shown from figure (3) for constant signal to noise ratio, probability of detection decreased for increasing of probability of false alarm. To remain the same probability of detection for different probability of false alarm, the SNR must be increased for decreasing of probability of false alarm.

\section{Performance of FAR With Spot Noise Jamming}

Assume the spot noise jammer with bandwidth $B_{j}$ equal to radar bandwidth $B_{r}$ [2-3] This means that it covers only a single channel of FAR band. Jammer will jam the victim radar only when radar chooses that jammed band, as shown in figure (4). It is clear, that the frequency agile radar has the self-immunity (ECCM) due to its ability to change its carrier frequency.

The effect of spot noise jamming on it can be derived from analysis of the pattern of frequency agility generation. Assuming that all the operating are equal likely to be used in the radar, the probability of jammer success in affecting radar is given by 
jamming's success probability $P(J)=1 / v$ and the target detection probability of frequency agile radar can be expressed as

$$
P_{d}=P(D / J) P(J)+P(D / N)(1-P(J))
$$

Where $P(D / J)$ is the probability of detection in the presence of jamming and noise (Interference), $P(D / N)$ is the probability of detection in the presence of noise alone.

$$
\begin{aligned}
& P(D / J)=Q(V-\sqrt{S I R}) \\
& P(D / N)=Q(V-\sqrt{S N R})
\end{aligned}
$$

Where SIR is the signal to interference ratio.

From Eq. (8), Eq. (9), and Eq. (10) the $P_{d}$ can be rewritten as

$$
P_{d}=\frac{1}{v} Q(V-\sqrt{S I R})+\left(1-\frac{1}{v}\right) Q(V-\sqrt{S N R})
$$

Figure (5) shows dependence of $P_{d}$ on the SNR for FAR for $P_{f a}=10^{-2}, \mathrm{JNR}=10 \mathrm{~dB}$, $v$ (number of channels) $=5$.

It is clear that the probability of detection in case of spot noise jamming for the case of FAR will be decreased slightly than the case of FAR without jamming in figure (3) because of $P(J)=20 \%$.

\section{FAR Performance in Case of Barrage Noise Jamming}

In this case the jammer try to increase its effectiveness by spreading its power to cover all the operating frequencies as shown in the Figure (6). The corresponding jamming success probability $P(J)$ is $100 \%$. This technique has a limited effect against the FAR, because it requires too much power in order to achieve an effective jamming [2-3].

Since the MF receives within its bandwidth $B_{r}$, only. The improvement in system performance due to frequency agility will be the ratio between the barrage jamming bandwidth and the MF bandwidth. So the $P_{d}$ can be rewritten as

$$
P_{d}=Q(V-\sqrt{\operatorname{SIR}})
$$


Figure (7) shows the effect of barrage noise jamming on the fixed frequency radar performance. Comparing the $P_{d}$ in the figure (5) and figure (7), it is clear that the barrage noise jamming reduce detection probability much more than the case of spot noise jamming at small SNR, but at high SNR the situation is reversed.

\section{Optimum Management of Jammer Power}

Two jamming techniques are proposed in order to improve the utilization of the available jamming power. These two techniques are: the partial band jamming and the linear frequency modulation (LFM) jamming technique (comb jamming), aim to maximize the degradation of the FAR performance due to the jamming, i.e. for a given jammer power to minimize the probability of detection of FAR.

\section{V.1 Partial band jamming}

Partial band jamming means that the jammer covers only a part, a fraction, of the FAR band. This means that jamming covers only a number of the frequency channels while the others suffer no jamming. Partial band jamming does not depend on which the channels to be jammed, if the probability of using channels is uniformly distributed. So it decreases the improvement gained by agility because of the increase in the jamming success probability $P(J)$ and the jamming power density $\zeta$, Figure (8) demonstrates the partial band jamming idea. In figure (8.a) shows the case of barrage jamming with power $P_{j}$ and figure (8.b) is for partial band jamming with the same total power. It is clear that the jamming density in the later case is better. By applying the partial band jamming, the jamming power density $\zeta_{j}$, of the jammed channels would increase while the remaining channels will operate without jamming. So a selection has to be considered; either to increase jamming power density or to increase probability of jamming.

The $P_{d}$ of a FAR under a barrage jamming and spot jamming are plotted in figure(9) Comparing the two curves it is clear that partial band jamming is better than barrage jamming at high SNR, and vise versa. This is evident because at low SNR, jamming power density is high with respect to the signal level so it is effective but as SNR increases the jamming power density, which is small, becomes not effective. In the later case it is required to concentrate jamming power in only a part of the FAR band to increase jamming power density, and insure the effectiveness of jamming on this part of the radar agility frequency band $\left(\beta_{t}\right)$.

\section{V.2 Comb jamming}

Comb jamming is a barrage noise jamming such that its frequency is generated by the linear frequency modulation of a narrow band noise such that it covers the required band of frequency. In this section mathematical expressions and analysis prove the enhancement in the jamming power effectiveness when using this technique. Consider the frequency modulation FM is varied linearly with time that, 


$$
f(t)=a\left(\frac{t}{T}\right), \quad-\frac{T}{2} \leq t \leq \frac{T}{2}
$$

The modulated signal can be written in general form in frequency domain as [1]

$$
\Phi_{F M}(w)=A T \sqrt{\frac{2 \pi}{b T}} \operatorname{Re}\left\{e^{-j w^{2} T /(2 b)}\left[\frac{\left[C\left(x_{2}\right)+C\left(x_{1}\right)\right]+j\left[S\left(x_{2}\right)+S\left(x_{1}\right)\right]}{\sqrt{2}}\right]\right\}
$$

Where $b=w_{\max } .-w_{\min }$.

$$
\begin{aligned}
& C(x)=\int_{0}^{x} \cos \left(\frac{\pi}{2} \xi^{2}\right) d \xi \\
& S(x)=\int_{0}^{x} \sin \left(\frac{\pi}{2} \xi^{2}\right) d \xi
\end{aligned}
$$

For large $x$

$$
\lim _{x \rightarrow \infty} C(x)=\frac{1}{2} \text {, and } \lim _{x \rightarrow \infty} S(x)=\frac{1}{2}
$$

Figure (11) shows the magnitude of the LFM signal. In the actual case when considering the carrier frequency $\left(\mathrm{f}_{0}\right)$, this spectrum is shifted to right along the frequency axis and is centered about the $f_{0}$. When choosing the spectrum width $2 b$ to be equal to the FAR band width and the carrier frequency $f_{0}$ to be equal to the mid point of this band, the spectrum will completely covers the FAR band

Let us define an infinite duration signal which is frequency modulated by a repetitive saw tooth signal with repetition interval $T$. This signal is expressed as

$$
y_{H M}(t)=\sum_{i=1}^{N} \phi_{H M}(t-i T)
$$

The repetition in time domain leads to discrete spectrum in the frequency domain and so $Y_{F M}(f)$ can be expressed as

$$
Y_{F M}(f)=\Phi_{F M}(f) \sum_{i=0}^{N} \delta\left(f-\frac{2 \pi i}{T}\right)
$$

Figure (12) gives the magnitude of the spectrum of a repeated linear frequency modulation (LFM) signal. In such case, the jamming power will be distributed on 
these lobed spectral lines only. Practically the spectral lines in figure (12) have a small bandwidth because FM modulation is not for single frequency but it is done to narrow band of noise. If the sweep period $T$ is selected to be equal to $1 / \Delta f$ ( $\Delta f$ is the frequency separation between each two successive frequencies of FAR) the spectrum of jamming signal will be presented each $\Delta f$, i.e. the spectrum of jamming signal will be presented only inside the matched filter and there will not be any power losses between the channels due to the redistribution of the jamming power spectrum. In case of barrage jamming, jamming power within this band is uniformly distributed all over the FAR band.

In case of barrage jamming, jamming power within this band is uniformly distributed all over the FAR band.

$$
\Delta f=\frac{\beta_{i}}{v}
$$

If the radar receiver received two jamming signals one of them is barrage jamming and the other is LFM jamming, the output of the matched filter will depend on the MF bandwidth $B_{r}$, if MF bandwidth equal to the separation between the successive channels which is equal to sweeping frequency $(1 / T)$ in the comb jamming, there is no difference between the two jamming types. If the MF bandwidth is less than the $\Delta f$, a part of the barrage noise will not received by the MF, MF output due to the comb jamming input will be more than the barrage jamming case by a ratio equal to separation between the successive frequencies to the MF bandwidth. The obtained gain when using comb jamming can be expressed as

$$
G=\frac{1 / T}{B_{r}}=\frac{1}{T B_{r}}
$$

Figure (13) shows the probability of detection against $\mathrm{SNR}$ at $\mathrm{P}_{\mathrm{fa}}=10^{-2}, J N R=10 \mathrm{~dB}$, $v=5$. The jamming signal is comb jamming. In addition the $\mathrm{P}_{\mathrm{d}}$ of a FAR under a comb jamming a barrage jamming plotted in solid line on figure (13). By comparing the $P_{d}$ in the figure (13), it is clear that the probability of detection in case of comb jamming has less detection probability than the case of barrage noise jamming. LFM enhance jamming effect on the radar performance on average by $13 \%$.

Figure (14) shows a comparison between the next five cases:

- FAR without jamming plotted in solid line.

- FAR with spot noise jamming plotted in dashed lines.

- FAR with partial band jamming $(60 \%$ jammed) plotted in dash-dot line.

- FAR with barrage jamming plotted in dot line.

- FAR with LFM jamming plotted in stares line. 


\section{Simulation of the Behavior of FAR}

In the simulation, FAR with ten channels is considered. The operating channel is selected randomly

-The degradation in the detection behavior of FAR in presence of spot noise jamming, with $\mathrm{JNR}=0 \mathrm{~dB}$ and occupies $10 \%$ of the operating radar agility band is shown in figure (15). One can see that the way to substitute the effect of jamming in FFR requires doubling the signal power while only slightly increase in signal power in FAR is sufficient to substitute the jamming effect. The effect of increasing jamming power on the behavior of FAR is plotted in figure (16). It is clear that FAR has a good performance compared to FFR due to the change of its operating frequency, i.e.it has a probability to operate at un-jammed rejoin. In FAR one dB increment in signal level is sufficient to substitute the effect of jamming.

-The jamming power used in the case of spot noise jamming case is spreaded to the total FAR bandwidth to simulate the barrage jamming. The number of jammed channels equals the total numbers of operating channels (10 channels) Figure (17) shows the effect of barrage noise jamming against FAR with different JNR. As shown from the simulated curves the performance of the radar degraded with the increasing of the jamming power level. However, it must be noted that due to spreading the jamming power over the 10 channels while the effective jamming power is reducing by a factor 0.1 . Thus there is a loss $10 \mathrm{~dB}$ of jamming power compared to the spot jamming case.

-The case of partial-band jamming is simulated by controlling of the filter bandwidth in the jamming signal generation site. The simulation study the case of JNR $=10 \mathrm{~dB}$, effect on $40 \%$ and $70 \%$ of the agility bandwidth. Figure (18) shows the performance of FAR with partial band jamming on $40 \%$ and $70 \%$. Extending the jamming signal bandwidth degrades the behavior of FAR. Finally figure (19) shows the results for different numbers of jammed channel, at JNR = $10 \mathrm{~dB}$. It is clear that the barrage jamming ( $100 \%$ jamming) gives the best effect for the SNR less than or equal $13 \mathrm{~dB}$. Spot jamming ( $10 \%$ jamming) becomes more effective for SNR more than $17 \mathrm{~dB}$. For the SNR between 13 and $17 \mathrm{~dB}$, the partial band jamming $40 \%$ and $70 \%$ gives higher effectiveness than both the barrage and spot jamming

-A LFM signal is used as a jamming technique the effective LFM jamming power will be more than the case of barrage jamming by the factor " $G$ ". In the simulation the ratio " $G$ " is taken to be " 2 ", which mean that the MF bandwidth is $1 / 2$ the minimum separation between two successive frequency component. Figure (20) shows the detection performance of a FAR when LFM jamming is used. Different jamming power levels used, that $J N R=0,5,10,15 \mathrm{~dB}$ while maintaining the $P_{f a}=10^{-2}$. More over, figure (21) shows a comparison between the use of barrage, comb, and $70 \%$ partial band jamming at the same jamming power level JNR $=10 \mathrm{~dB}, \mathrm{P}_{\mathrm{ta}}=10^{-2}$. As shown the comb jamming has higher effect and produces the largest reduction in the probability of detection. 


\section{Conclusion}

Analysis of results shows the high immunity of FAR against spot noise jamming. The probability of jamming power interception by radar receiver is inversely proportional to the number of possible frequency channels. Although the spot noise jamming seems to be not effective against the FAR but during the analysis it is found to be effective at higher SNR (short ranges).

Barrage noise jamming is more effective than spot noise jamming. This is because of the $100 \%$ jamming interception probability. This is clear for low SNR (long target range), but at higher SNR (short target ranges) the spot noise jamming will have a higher effect

Partial band jamming is a proposed approach for optimum utilization of the jamming power. Obtained results show that increasing the number of jammed channels makes jamming power more effective on the FAR at low SNR (long target ranges), but at high SNR (short ranges) the effect will be reversed.

For the second proposed approach, the linear frequency modulation (LFM) jamming, the improvement of jamming effectiveness against FAR depends on the ratio (G) of the separation between the successive frequencies to the MF bandwidth. Barrage jamming corresponds to the ratio $\mathrm{G}=1$.

\section{References}

1- Peyton Z. peebles, JR., Radar Principles, John Wiley and Sons, Inc., 1998

2- Leroy B. Van Brunt, Applied ECM,vol.I, EW Engineering, Inc. 1978.

3- D. Curtis Schleher, Introduction to Electronic Warfare, Artech House, INC 1986. 


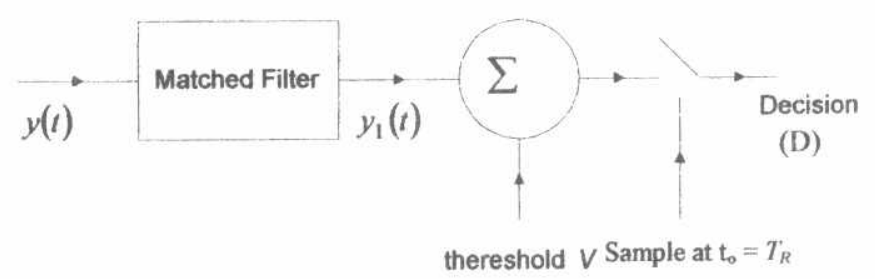

Figure (1) the matched filter equivalent processor.

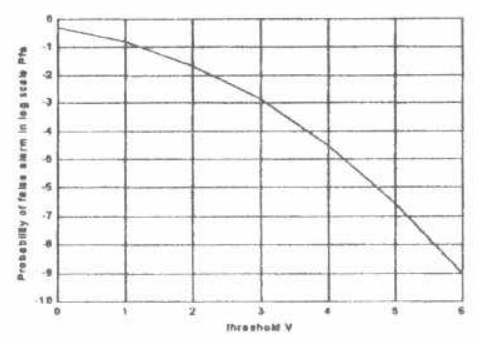

Figure (2) The dependence of $P_{f a}$ on the threshold level $V$ for the case of white noise.

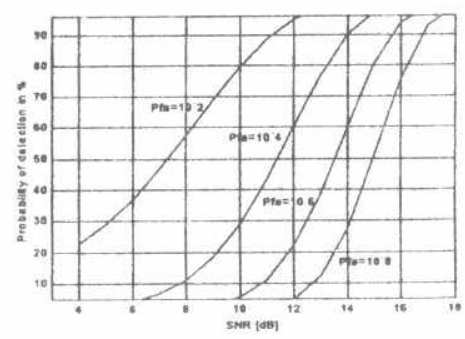

Figure (3) $P_{d}$ for exactly known signal in white noise.

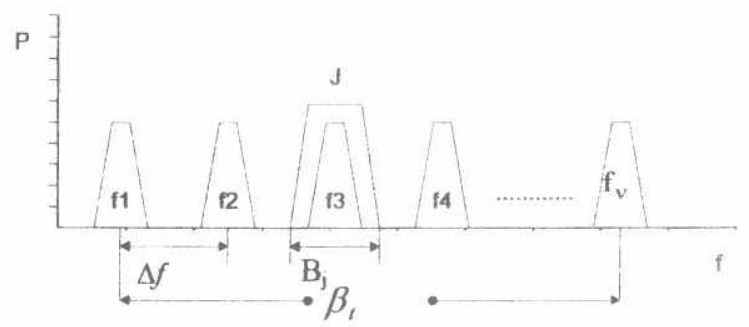

Figure (4) JNR power spectrum at the radar, Agile Frequency radar. 


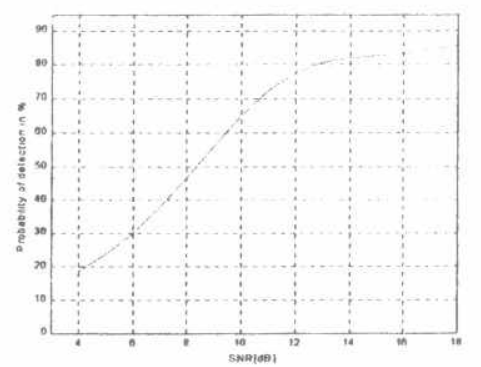

Figure (5) Dependence of $P_{d}$ on SNR, $P_{f a}=10^{-2}$.

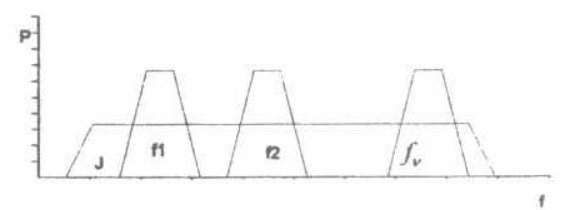

Figure (6) JNR power spectrum at the radar, FAR, Barrage jamming.

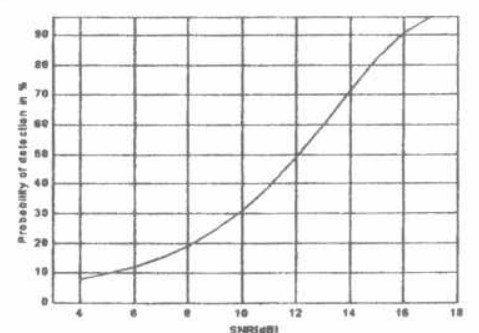

Figure (7) the dependence the Probability of detection as a function of SNR in case of barrage jamming. For $J N R=10 \mathrm{~dB}, \mathrm{P}_{\mathrm{fa}}=10^{-2}, \& \mathrm{~B}_{\mathrm{j}} / \mathrm{B}_{\mathrm{r}}=5$.

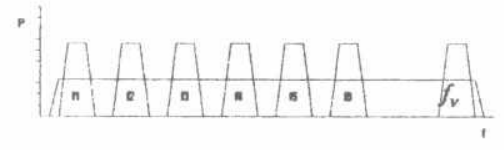

Figure (8,a) JNR power spectrum at the radar, FAR, Barrage jamming.

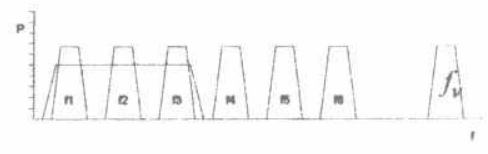

Figure (8.b) JNR power spectrum at the radar, FAR, and Partial band jamming. 


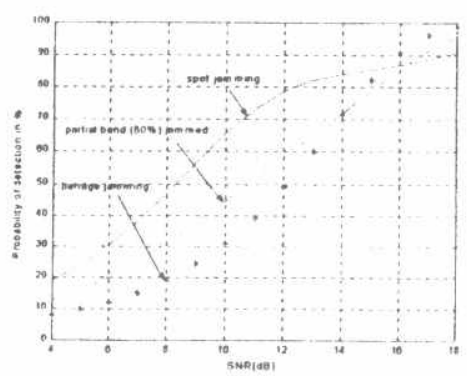

Figure (9) Probability of detection at JNR $=10 \mathrm{~dB}$, partial band jamming, FAR $(v=5)$, jamming $60 \%$.

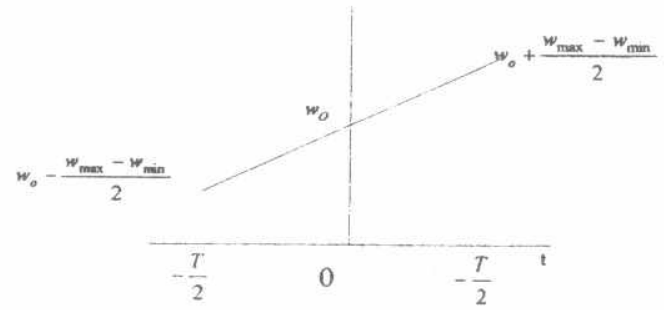

Figure (10) Linear FM.

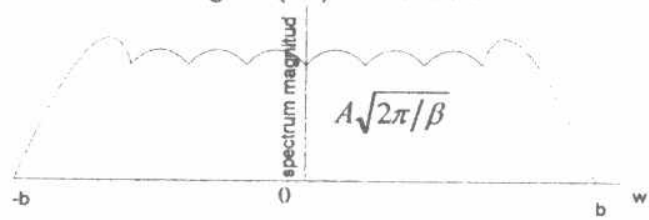

Figure (11) Spectrum magnitude of Linear FM signal.

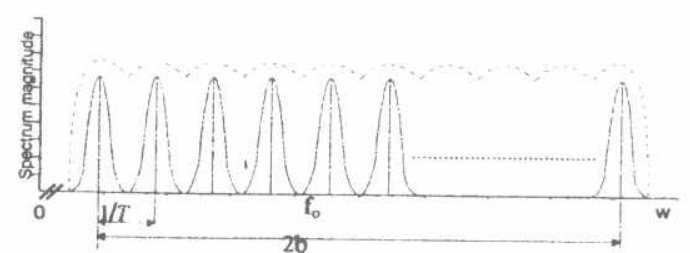

Figure (12) Spectrum magnitude of Linear FM signal, repetitive. 


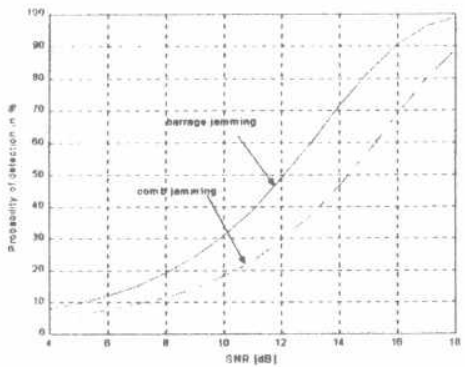

Figure (13) Probability of detection at JNR $=10 \mathrm{~dB}$, comb jamming, $\operatorname{FAR}(v=5)$.

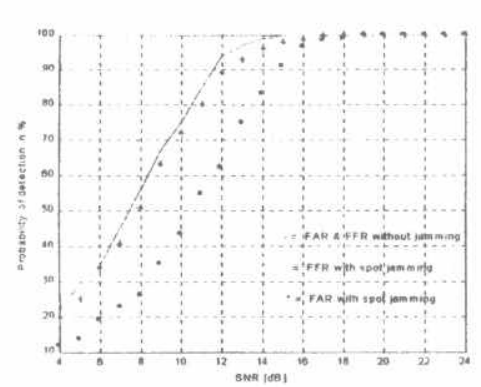

Figure (15) Simulated curves for FFR, FAR with spot jamming.

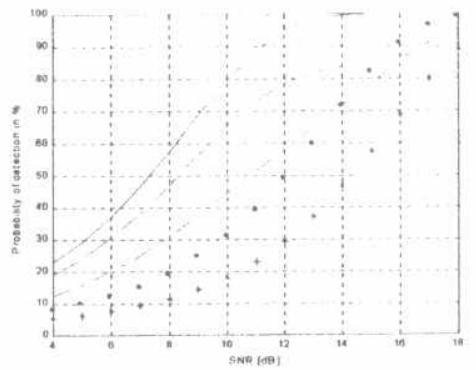

Figure (14) FAR behavior without, and with different types of iamming.

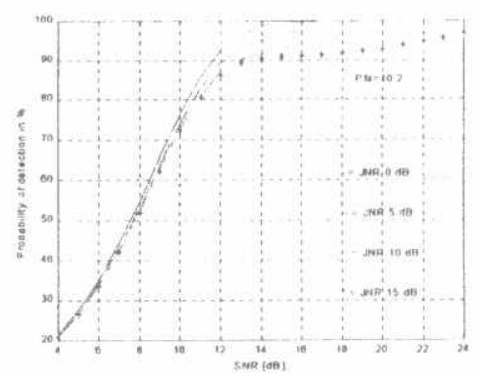

Figure (16) FAR with spot jamming at different JNR.

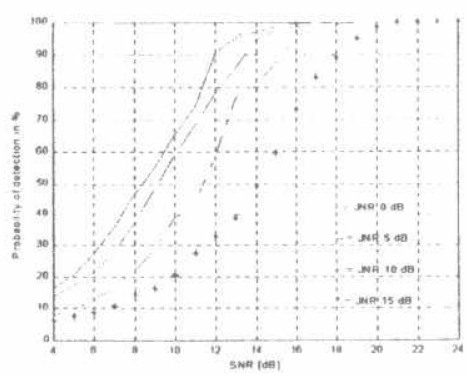

Figure (17) Simulated performance of FAR with barrage jamming, $P_{t a}=10^{-2}$ 


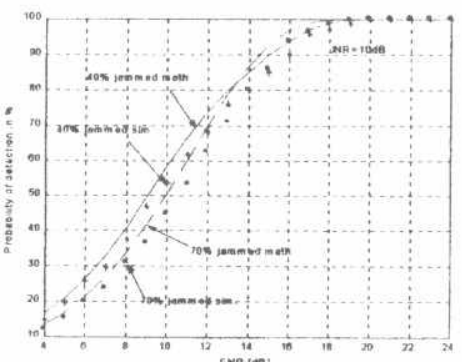

Figure (18) Effect of partial band jamming on the FAR.

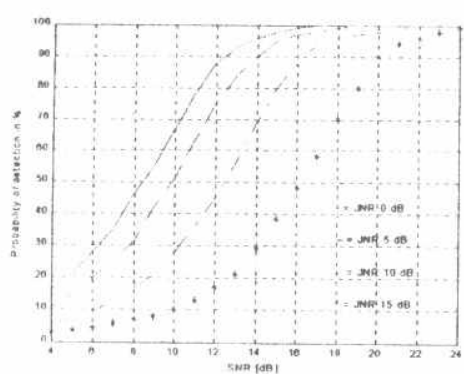

Figure (20) detection performance of FAR with comb jamming.

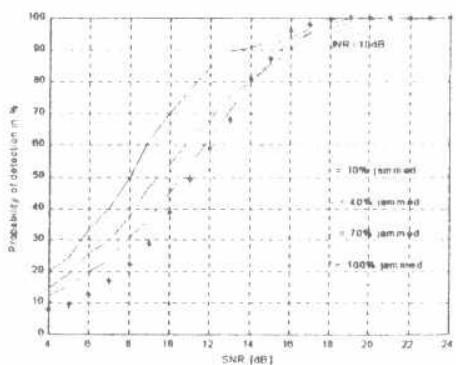

Figure (19) Simulated FAR performance with partial band jamming, $J \mathrm{NR}=10 \mathrm{~dB}, \mathrm{P}_{\mathrm{fh}}=10^{-2}$.

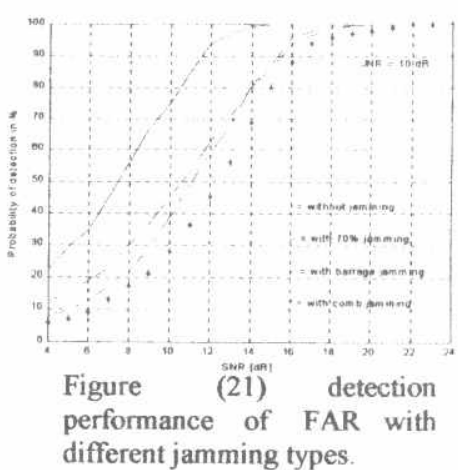

